\title{
The Effect of Infrared Light's Power on the Infrared Spectra of Thin Films
}

\author{
Giovanna Scarel ${ }^{*}$, Eric C. Stevens ${ }^{2}$ \\ ${ }^{1}$ Department of Physics and Astronomy, James Madison University, Harrisonburg, VA, USA \\ ${ }^{2}$ Department of Chemical and Biomolecular Engineering, North Carolina State University, Centennial Campus, \\ Raleigh, NC, USA \\ Email: *scarelgx@jmu.edu
}

How to cite this paper: Scarel, G. and Stevens, E.C. (2019) The Effect of Infrared Light's Power on the Infrared Spectra of Thin Films. World Journal of Condensed Matter Physics, 9, 1-21. https://doi.org/10.4236/wjcmp.2019.91001

Received: January 9, 2019

Accepted: January 31, 2019

Published: February 3, 2019

Copyright (c) 2019 by author(s) and Scientific Research Publishing Inc. This work is licensed under the Creative Commons Attribution International License (CC BY 4.0).

http://creativecommons.org/licenses/by/4.0/

(c) (i) Open Access

\begin{abstract}
There are numerous evidences that a relationship exists between the average power $P$ of electromagnetic waves and the mechanical motion of the objects interacting with them. We investigate the effects of infrared (IR) light's average power $P$ on the transmission Fourier transform infrared (FTIR) spectra of thin cubic $\mathrm{Yb}_{2} \mathrm{O}_{3}$ and rhombohedral $\mathrm{LaAlO}_{3}$ films deposited on silicon via atomic layer deposition. We find that different values of $P$ of the IR light displace the minima of the absorption bands. This effect is reproducible in different sets of experiments and in different spectrometers. To interpret the experimental findings, we use the law of conservation of energy. We find a correlation among the energy of the IR waves and the number, moment of inertia, and vibrational/rotational frequency of the bonds involved in the vibrational or rotational motion. The law of conservation of energy unveils that larger values of $P$ of the IR light and lower wavenumbers of the resonances involve a larger number of crystal bonds. One practical application of our approach is that it suggests a way to improve the sensitivity of the FTIR spectra of thin crystalline films in the far IR region.
\end{abstract}

\section{Keywords}

Infrared, Power, Vibrational Spectroscopy, Atomic Layer Deposition

\section{Introduction}

Absorption of electromagnetic (EM) waves may generate mechanical motion [1] and vibrations [2], at the molecular [1] [2] or nuclear [3] [4] levels. On the other hand, rotating objects can emit EM waves. For example, the magnetic fields on the sun's surface rotate by some angles while generating solar flares with emission at a wavelength, e.g., $656.28 \mathrm{~nm}$, which corresponds to the red $\mathrm{H} \alpha$ line [5]. 
Rotating galaxies seem to generate the [CII] line at wavelength $157.74 \mu \mathrm{m}$, which is in the microwave range of the EM spectrum [6]. Neutron star merger events radiate $\gamma$-rays plus radio waves [7]. Dust grains of variable size in space are expected to emit infrared (IR) light and microwaves in various types of interstellar environments [8].

The magnitude of the average power $P$ of the EM waves seems to play a key role in linking the magnitude of the mechanical motions to the properties of the EM waves generating them or caused by them. For example, in Thomson scattering a precise analytical expression exists between the power over area (i.e. the intensity $I_{0}$ ) of the absorbed laser beam with central frequency $\omega_{0}$, and the kinetic energy $\left(\varepsilon_{0}\right)$ and emission angle $\theta_{m}$ of the scattered electrons such that $I_{0} \propto\left[\left(\omega_{0} \varepsilon_{0}\right) f\left(\theta_{m}\right)\right]^{2}$ [3] [4], where $f\left(\theta_{m}\right)$ is a function of $\theta_{m}$. In the neutron star merger event GW170817 it was recently discovered that the $\gamma$-ray plus radio wave afterglow signals and the dynamical ejecta are emitted in the form of a choked jet cocoon [7]. Researchers drew this conclusion after studying the flux densities, i.e. the energy versus time profiles or power, of the emitted radio waves [7]. On a smaller scale, vibrational spectra enabled the investigation of the absorption of IR light in the formation of urethane and polyurethane. The vibrational spectra unveiled that the addition of a vibrational excitation to thermal energy accelerated the reaction rate of formation [2]. From the information that the IR light was emitted with an energy per pulse of $3 \mu \mathrm{J}$, repetition rate of $2 \mathrm{kHz}$, and wavelength of $2857 \mathrm{~nm}$, we estimated that the average power of the laser was $P=6 \mathrm{~mW}$. At this value of $P$, approximately 1190 reactions producing urethane and 2976 reactions producing polyurethane were activated within one period $\tau=9.5 \mathrm{fs}$ of the illuminating IR light. Finally, at the nanoscale level, a linear relationship was found between the rotational frequency of an optically trapped dielectric particle and the power of the trapping laser [9]. According to the authors of Ref. [9], however, the dominating torque transfer mechanism in this experiment is still unknown.

To understand the role of the light's average power $P$ in generating mechanical motion in light-matter interaction involving atoms in crystalline bonds, we consider the wave nature of light. Indeed, for light as a wave, the power over area (i.e. the intensity $I_{0}$ ) is the Poynting vector, and the amount of energy conserved in the interaction of light with matter is $P \tau$, where $\tau$ is the period of the of the light wave. These concepts and their consequences in various natural phenomena are discussed in Ref. [10]. In this article we specifically investigate the effects of the average power $P$ on the transmission Fourier transform IR (FTIR) [11] spectra of thin crystalline cubic $\mathrm{Yb}_{2} \mathrm{O}_{3}$ and rhombohedral $\mathrm{LaAlO}_{3}$ films deposited on silicon (Si). We first present the method used to grow the thin films via atomic layer deposition (ALD) [12] [13], and report the properties of the thin films. We then describe how we obtained the transmission FTIR spectra [11] of the thin films, focusing in particular on how we achieved the different values of the average power $P(25 \mathrm{~mW} \pm 5.2 \mu \mathrm{W}, 16.4 \mathrm{~mW} \pm 4.5 \mu \mathrm{W}, 12.1 \mathrm{~mW} \pm 5.1 \mu \mathrm{W}$, and 7.9 
$\mathrm{mW} \pm 4.9 \mu \mathrm{W})$. In the Result Section we show that different values of the average power $P$ displace the location (in wavenumber, or frequency in units of $\mathrm{THz}$ ) of the minima of the absorption bands in the FTIR spectra of the thin films. We ascribe this phenomenon to the fact that the average power $P$ affects the vibrational energy of the crystalline structure of the thin films, $E_{v i b}$, which, in turn, determines the location of the minima of the absorption bands in the FTIR spectra. In the Discussion Section we link the average power $P$ to the location of the minima of the absorption bands observing that the moment of inertia $I$ and the vibrational frequency $\omega$ of the vibrating bond depend on $E_{v i b}$. In turn, $E_{v i b}$ is correlated to $P \tau$, the amount of energy conserved in the interaction of light with matter. Since $E_{v i b}$ represents the vibrational/rotational energy of the whole crystal structure involved in the interaction with the IR light, this energy depends on the number $\eta$ of the interested bonds. Therefore, using a model based on the law of conservation of energy [10], and knowing $P, \tau, E_{v i b}, \omega$ and $I$, we estimate the number $\eta$ of the bonds involved in the interaction between the IR light and the thin crystalline films. To highlight the significance of our results we report the outcome of our literature search showing that other researchers acknowledged the existence of displacements in the minima of the absorption bands in FTIR spectra [14]. These authors, however, were not able to justify such displacements. We also found that the existence of a relationship among $P, E_{v i b}, \omega$, and $I$ is acknowledged, e.g., in the motion of a dielectric particle optically trapped by a laser [9]. This finding not only supports our approach, but also underlines its innovative character in the field of FTIR spectroscopy. In the Conclusions Section we outline the significance of our study. First we highlight that $\eta$, the number of bonds involved in the interaction between the IR light and the thin crystalline films, might represent a "classical" way (as opposed to the usual "quantum" way) to count the number of the quasi-particles usually called phonons, through the link among $P, \tau, E_{v i b}, \omega, I$, and $\eta$. As far as the practical application of our findings are concerned, our method suggests that, by increasing the power of the IR light, it should be possible to enhance the sensitivity of the FTIR spectra of thin crystalline films in the far IR region. Achieving this goal is possible due to the direct proportionality between moment of inertia and average power, i.e. $I \propto P$, as we have found in our study. Applying our strategy, however, requires keeping in mind that such increase in sensitivity is accompanied by a slight shift in location of the minima of the absorption bands.

\section{Experimental Methods}

We analyzed two different types of thin oxide films using transmission FTIR spectroscopy at four average power levels of the IR light using two different spectrometers. In one of the two spectrometers the original power of the source was lowered using a polarizer and a copper mesh. The details of the materials used and measurements performed are as follows. 


\subsection{Thin Films}

Uniform thin cubic $\mathrm{Yb}_{2} \mathrm{O}_{3}\left(500 \mathrm{~nm}\right.$, thick) and rhombohedral $\mathrm{LaAlO}_{3}$ films (550 $\mathrm{nm}$ thick) on Si were prepared via ALD [12] [13] with growth parameters and precursors described in Ref. [15]. The films exhibit remarkable stability [13] as they retain their characteristics over more than the year during which we collected and analyzed their transmission FTIR spectra. We assessed the crystallinity of the thin films using far-IR FTIR spectroscopy, as shown in Appendix 1.

\subsection{FTIR Spectrometers}

We investigated the effects of different levels of the IR light's average power $P$ on transmission FTIR spectra using a $\mathrm{N}_{2}$-purged Bruker Vertex 70 spectrometer with a Q301 globar source emitting at $P=25 \mathrm{~mW} / \mathrm{cm}^{2}$, and a $\mathrm{N}_{2}$ purged Nicolet Magna 6700 spectrometer with a globar source emitting at $P=12.1 \mathrm{~mW} / \mathrm{cm}^{2}$. On the Bruker Vertex 70 spectrometer we achieved $P=16.4 \mathrm{~mW} / \mathrm{cm}^{2}$ and $P=7.9 \mathrm{~mW} / \mathrm{cm}^{2}$ by inserting a copper mesh or polarizer (PIKE Technologies 090-1000 ZnSe polarizer), respectively, in the beam path on the sample holder in front of the thin film. For the measurements with the polarizer we selected p-polarization (or TM-polarization). We investigated the features of the thin cubic $\mathrm{Yb}_{2} \mathrm{O}_{3}$ and rhombohedral $\mathrm{LaAlO}_{3}$ films in the middle IR range (MIR, $350-$ $7,500 \mathrm{~cm}^{-1}$ wavenumber, $28,600 \mathrm{~nm}-1300 \mathrm{~nm}$ wavelength or $95.33 \mathrm{fs}-4.33 \mathrm{fs}$ period). In both spectrometers and in all measurements we selected a resolution of $4 \mathrm{~cm}^{-1}$ and collected the spectra with 100 scans. In both spectrometers, we positioned the thin film on a vertical transmission sample holder perpendicular to the IR beam. We explored only normal incidence transmission spectra because one of the two spectrometers is equipped with neither a reflection nor a rotating transmission accessory. The lack of a reflection accessory on one of the spectrometers prevented us from studying films on metal substrates, which do not enable transmission studies. We measured the spectra with the Bruker Vertex 70 spectrometer two days before measuring them with the Nicolet Magna 6700 spectrometer to allow time for the trip from Harrisonburg (VA) to Raleigh (NC) where the two spectrometers are located. We kept the samples sealed and protected during the transfer from one location to the other, and between all sets of measurements. The collected spectra exhibit excellent reproducibility demonstrating the high stability of the thin crystalline films we used in this research [13]. We discuss the effects of the choice of the Si substrate in Appendix 2 and Appendix 3.

\subsection{Power Measurements}

We measured the power of the IR light before all measurements using a power-meter sensor Coherent PowerMax RS PS19, sensitive to wavelength and average power ranges of $300-11,000 \mathrm{~nm}$ and $100 \mu \mathrm{W}$ to $1 \mathrm{~W}$, respectively. The absorption bands of interest in the examined thin films appear in the $470-950$ $\mathrm{cm}^{-1}$ wavenumber range (or 10,526 - 21,276 $\mathrm{nm}$ wavelength range). For the ab- 
sorption bands of the cubic $\mathrm{Yb}_{2} \mathrm{O}_{3}$ films, which appear in the $840-950 \mathrm{~cm}^{-1}$ wavenumber range $(10,520-11,905 \mathrm{~nm}$ wavelength range), the IR light's power estimation is within the range of sensitivity of the power sensor. On the other hand, the absorption bands of the rhombohedral $\mathrm{LaAlO}_{3}$ films appear in the 470 $560 \mathrm{~cm}^{-1}$ wavenumber range $(21,276-17,857 \mathrm{~nm}$ wavelength range), which is outside the operating range of the power sensor. Therefore, in the case of the rhombohedral $\mathrm{LaAlO}_{3}$ films the estimated power is affected by a systematic error. This systematic error, however, does not prevent us from evaluating the shifts of the minima in the absorption bands observed with either the Bruker Vertex 70 or the Nicolet Magna 6700 spectrometers.

\section{Nomenclature}

To simplify reading the Results and Discussion Sections, here we list the meaning of the most significant variables presented: $P$ average power of the IR light, $\tau$ period of the IR light, $E_{v i b}$ vibrational energy of the crystalline structure of the thin films, $\omega$ the vibrational frequency of the vibrating bond, $I$ moment of inertia of the vibrating bond, $\eta$ number of bonds involved in the interaction between the IR light and the thin crystalline films, $m_{a t}$ mass of the atoms involved in each bond, and llattice parameter of the film's crystalline structure.

\section{Results}

Figure 1 displays the transmission FTIR spectra of the cubic $\mathrm{Yb}_{2} \mathrm{O}_{3}$ films on $\mathrm{Si}$ and rhombohedral $\mathrm{LaAlO}_{3}$ films on $\mathrm{Si}$ collected with IR light's average power $P=12.1 \mathrm{~mW}$ and $25 \mathrm{~mW}$. First, we observe that the transmission level is about $50 \%$ lower for the spectra acquired with $P=12.1 \mathrm{~mW}$ (panels (a) and (c)). Additionally, the absorption bands for both the $\mathrm{Yb}_{2} \mathrm{O}_{3}$ and $\mathrm{LaAlO}_{3}$ films on Si are deeper and better defined when collected with IR light at an average power $P=25 \mathrm{~mW} \quad$ (panels (b) and (d)). Furthermore, a careful inspection of Figure 1 indicates that the location of the minima of the absorption bands are different in the spectra obtained with the Bruker Vertex $70(P=25 \mathrm{~mW})$ and the Nicolet Magna 6700 ( $P=12.1 \mathrm{~mW}$ ) spectrometers.

Figure 2 and Figure 3 compare the effects of different magnitudes of the average power $P$ of the IR light on the FTIR spectra of the cubic $\mathrm{Yb}_{2} \mathrm{O}_{3}$ and the rhombohedral $\mathrm{LaAlO}_{3}$ films on $\mathrm{Si}$, respectively. Differently than in Figure 1, to obtain Figure 2 and Figure 3 we changed the average power $P$ of the IR light only on the Bruker Vertex 70 spectrometer by either inserting or omitting a copper mesh or a polarizer in the IR beam path. This method enabled us to achieve $P=7.9 \mathrm{~mW}, P=16.4 \mathrm{~mW}$ and $25 \mathrm{~mW}$. First, we note that Figure 2(a), for the cubic $\mathrm{Yb}_{2} \mathrm{O}_{3}$ films, and Figure 3(a), for the rhombohedral $\mathrm{LaAlO}_{3}$ films, show that the transmission levels vary only within $15 \%$ for three different average power magnitudes, while the depth of the absorption bands do not exhibit significant variations. We can thus speculate that the large variations in the transmission levels and the depth of the absorption bands in Figure 1 can be ascribed 

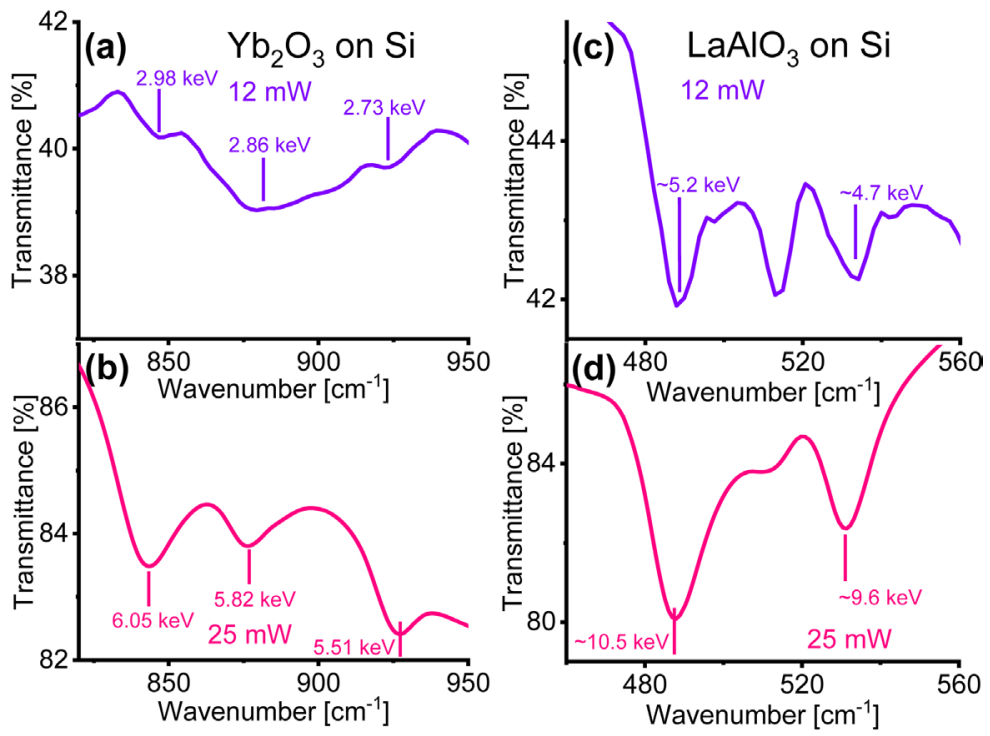

Figure 1. Transmission Fourier transform infrared (FTIR) spectra of cubic $\mathrm{Yb}_{2} \mathrm{O}_{3}$ films on $\mathrm{Si}$ acquired with infrared (IR) light's power of (a) $12.1 \mathrm{~mW}$ and (b) $25 \mathrm{~mW}$. FTIR spectra of rhombohedral $\mathrm{LaAlO}_{3}$ films on Si collected with IR light's power of (c) 12.1 $\mathrm{mW}$ and (d) $25 \mathrm{~mW}$. The values of $P \tau$ are labelled in units of $\mathrm{keV}$ for the relevant absorption bands in all panels. These values are approximated for the case of the $\mathrm{LaAlO}_{3}$ films on Si in panels (c) and (d) since the spectral features appear in a range located at the edge of the sensitivity of the power sensor used in this research.
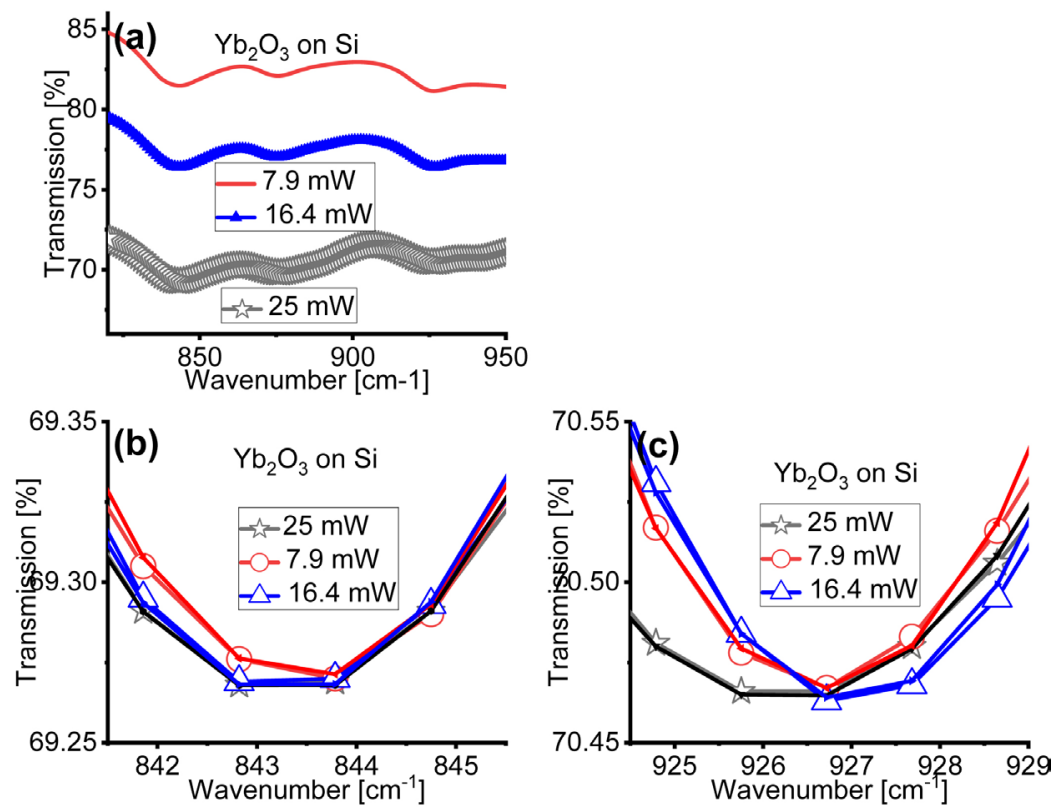

Figure 2. Transmission Fourier transform infrared (FTIR) spectra of cubic $\mathrm{Yb}_{2} \mathrm{O}_{3}$ films on $\mathrm{Si}$ acquired with infrared (IR) light's power of $7.9 \mathrm{~mW}, 16.4 \mathrm{~mW}$ and $25 \mathrm{~mW}$. Panel (a) shows the original transmission FTIR spectra in the wavenumber range where the relevant absorption bands appear. Panels (b) and (c) zoom into the location where the minima in the absorption bands of the FTIR spectra appear after offsetting the spectra so that they overlap. In panels (b) and (c) the experimental data are fitted by the hyperbolic secant function in Equation (1), which facilitates the precise determination of the location of the minima of the absorption bands, i.e. the parameter $w_{c}$. 

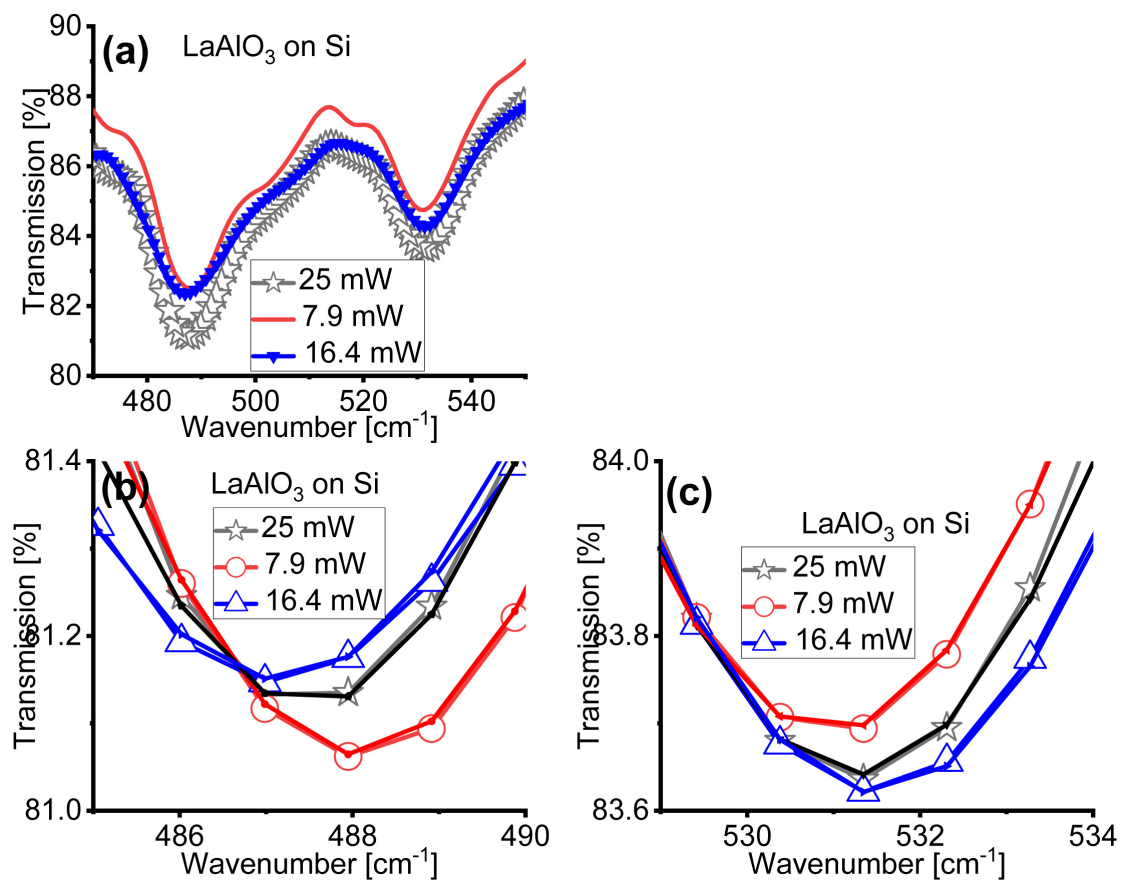

Figure 3. Transmission Fourier transform infrared (FTIR) spectra of rhombohedral $\mathrm{LaAlO}_{3}$ films on Si acquired with infrared (IR) light's power of $7.9 \mathrm{~mW}, 16.4 \mathrm{~mW}$ and 25 $\mathrm{mW}$. Panel (a) shows the original transmission FTIR spectra in the wavenumber range where the relevant absorption bands appear. Panels (b) and (c) zoom into the location where the minima in the absorption bands of the FTIR spectra appear after offsetting the spectra so that they overlap. In panels (b) and (c) the experimental data are fitted by the hyperbolic secant function in Equation (1), which facilitates the precise determination of the location of the minima of the absorption bands, i.e. the parameter $w_{c}$.

to the specific spectrometer used. Then, by examining Figure 2(b), Figure 2(c), Figure 3(b) and Figure 3(c), we again find that the location of the minima in the absorption bands shifts in response to different magnitudes of the average power $P$, as reported in Table 2 and in agreement with the trends reported in Figure 1 and Table 1. We thus infer that the location of the minima of the absorption bands is indeed related to the average power $P$ of the IR light. To carefully determine the location of the minima of the absorption bands, in Figure 2(b), Figure 2(c), Figure 3(b), and Figure 3(c) we fit the experimental transmission $(T)$ versus wavenumber $(w)$ spectra with a hyperbolic secant function:

$$
T(w)=T_{\text {off }}+T_{\text {osc }} \operatorname{sech}\left(\frac{w-w_{c}}{W_{c}}\right),
$$

where $T_{\text {off }}$ is the starting transmission level, $T_{\text {osc }}$ is the amplitude of the hyperbolic secant well, $w_{c}$ is the location of the minimum, and $W_{c}$ is the width of the hyperbolic secant well. We selected the hyperbolic secant function to fit the spectra because such a function overlaps the experimental data points and facilitates the process of locating the minimum of the well. The parameter $w_{c}$, i.e. the location of the minimum of the hyperbolic secant well, is identified as $\omega$ and reported in Table 1 and Table 2 for the minima of the significant absorption 
Table 1. For both the cubic $\mathrm{Yb}_{2} \mathrm{O}_{3}$ and the rhombohedral $\mathrm{LaAlO}_{3}$ lattices, we report the mass $m_{a t}$ of the atoms involved in the relevant bonds [16] [17], the lattice parameter 1 [18] [19], and the vibrational frequency $\omega$ at which we find the minima of the absorption bands in Fourier transform infrared (FTIR) spectra obtained at average power $P$ of $12.1 \mathrm{~mW}$ and $25 \mathrm{~mW}$. For the rhombohedral $\mathrm{LaAlO}_{3}$ films we choose an average value of the lattice parameter $l$ to simplify the calculations. The average vibrational frequencies $\omega$, or the parameter $w_{c}$ in Equation (1), are determined from the minima in the absorption bands of the FTIR spectra in Figure 1 using Equation (1) as fitting function.

\begin{tabular}{ccc}
\hline Mass $m_{a t}$ & Lattice parameter $l(\mathrm{~nm})$ & Frequency $\omega(\mathrm{THz})$ \\
\hline $\mathrm{Yb}_{2} \mathrm{O}_{3}: 27.23 \times 10^{-26} \mathrm{~kg}$ & 1.06 & 25.38 at $12.1 \mathrm{~mW}$ \\
& 25.31 at $25 \mathrm{~mW}$ \\
$\mathrm{LaAlO}_{3}: 15.61 \times 10^{-26} \mathrm{~kg}$ & 26.43 at $12.1 \mathrm{~mW}$ \\
& 26.30 at $25 \mathrm{~mW}$ \\
& 27.78 at $12.1 \mathrm{~mW}$ \\
& 27.81 at $25 \mathrm{~mW}$ \\
& 14.65 at $12.1 \mathrm{~mW}$ \\
& 14.62 at $25 \mathrm{~mW}$ \\
\hline
\end{tabular}

Table 2. For both the cubic $\mathrm{Yb}_{2} \mathrm{O}_{3}$ and the rhombohedral $\mathrm{LaAlO}_{3}$ lattices, we report the vibrational frequency $\omega$ at which we find the minima of the absorption bands in Fourier transform infrared (FTIR) spectra obtained at an average power $P$ of $25 \mathrm{~mW}, 16.4$ $\mathrm{mW}$ and $7.9 \mathrm{~mW}$ shown in Figure 2 for the $\mathrm{Yb}_{2} \mathrm{O}_{3}$ films and in Figure 3 for the $\mathrm{LaAlO}_{3}$ films. For simplicity, for the $\mathrm{Yb}_{2} \mathrm{O}_{3}$ films we display the values for only two out of the three relevant absorption bands. Also here, $\omega$ is the parameter $w_{c}$ in Equation (1).

\begin{tabular}{cccc}
\hline & $\begin{array}{c}\text { Frequency } \omega(\mathrm{THz}) \\
\text { at } 25 \mathrm{~mW}\end{array}$ & $\begin{array}{c}\text { Frequency } \omega(\mathrm{THz}) \\
\text { at } 16.4 \mathrm{~mW}\end{array}$ & $\begin{array}{c}\text { Frequency } \omega(\mathrm{THz}) \\
\text { at } 7.9 \mathrm{~mW}\end{array}$ \\
\hline $\mathrm{Yb}_{2} \mathrm{O}_{3}$ on $\mathrm{Si}$ & 25.299 & 25.299 & 25.304 \\
& 27.787 & 27.809 & 27.801 \\
$\mathrm{LaAlO}_{3}$ on $\mathrm{Si}$ & 14.625 & 14.614 & 14.641 \\
& 15.938 & 15.945 & 15.928 \\
\hline
\end{tabular}

bands identified in our thin films. An attentive examination of these tables suggests that the location of the minima of the absorption bands do not exhibit a monotonic trend with the magnitude of the average power $P$.

Figure 4 reports the FTIR spectra of the cubic $\mathrm{Yb}_{2} \mathrm{O}_{3}$ acquired on different days to assess the reproducibility of the location of the minima of the absorption bands. We observe that the location of the absorption bands at $843 \mathrm{~cm}^{-1}$ in Figure 4(a) and Figure 4(b), and $927 \mathrm{~cm}^{-1}$ in Figure 4(c) and Figure 4(d), are consistent in days 1 and 2. This finding enables us to conclude that, at a fixed magnitude of the average power $P$, the location of the absorption bands does not change, and helps us constraining to $P$ the responsibility of the changes in the 

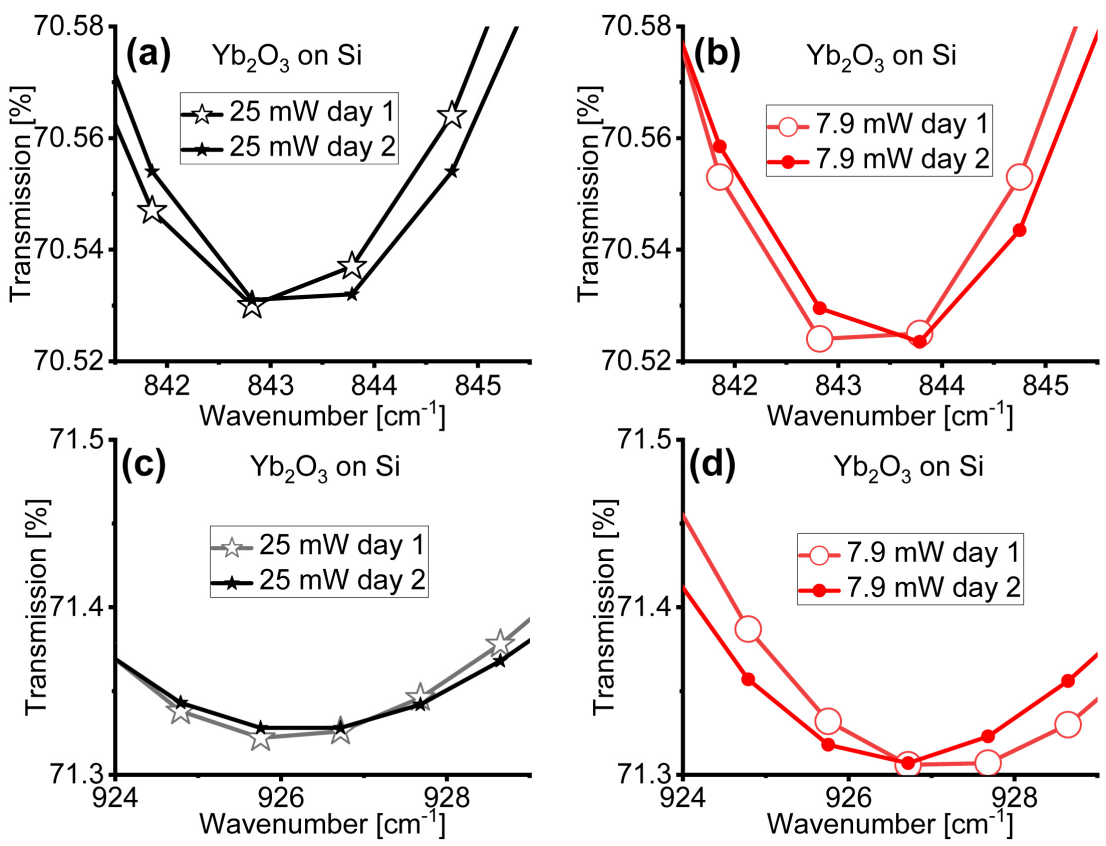

Figure 4. Fourier transform infrared (FTIR) spectra of cubic $\mathrm{Yb}_{2} \mathrm{O}_{3}$ films on Si acquired with infrared (IR) light's power of $25 \mathrm{~mW}$ (panels (a) and (c)) and $7.9 \mathrm{~mW}$ (panels (b) and $(\mathrm{d})$ ) in two different days (day 1 and day 2 ) to assess the reproducibility of the location of the minima of the absorption bands. In Appendix 6 we show the same spectra for the rhombohedral $\mathrm{LaAlO}_{3}$ films on Si.

location of the minima of the absorption bands. Instead, to highlight that the displacement of the minima of the absorption bands occurs uniquely in crystalline films, in Appendix 4 we unveil that the displacements in the location of the absorption bands does not to affect the molecular vibrations. To achieve this conclusion, we examine, for example, the region of the carbon residues in the $2,500 \mathrm{~cm}^{-1}$ wavenumber region: here we do not observe any absorption band displacement. Finally, in Appendix 5 we rule out the possibility that sample heating causes the displacement of the location of the minima of the absorption bands.

In summary, our experimental FTIR spectra highlight two major effects induced by varying the magnitude of the average power $P$ of the IR light: 1 ) the displacement of the minima of the absorption bands of the crystalline thin films and 2) the lack of a monotonic trend in the extent and direction of the displacements.

\section{Discussion}

We argue that the origin of the displacements and of their lack of a monotonic trend in extent and direction is linked to the magnitude of $P \tau$, where $P$ is the average power and $\tau$ the period of the IR light. We start our argument by recalling that the energy $P \tau$ is the amount of energy conserved in the interaction between the IR light and the thin crystalline films on Si [10]. The values of $P \tau$ are labelled in units of $\mathrm{keV}$ for the relevant absorption bands in all panels of Figure 1. These values are larger for the absorption bands in the spectra col- 
lected with $P=25 \mathrm{~mW}$, i.e. for the larger values of $P$ we used, and for the $\mathrm{LaAlO}_{3}$ films, i.e. for the larger values of the period $\tau$ or lower values of the wavenumber at which we detected in the absorption bands. We thus hypothesize that the energy $P \tau$ transfers vibrational energy $E_{v i b}$ to the bonds of the thin crystalline films illuminated by the IR light. We correlate $P \tau$ and $E_{v i b}$ through the law of conservation of energy as follows:

$$
P \tau=\eta \frac{1}{2} I \omega^{2}=\eta E_{v i b}
$$

In Equation (2), $I=m_{a t} l^{2}$ is the moment of inertia of $\mathrm{Yb}-\mathrm{O}$ [16], La-Al or Al-O bonds [17]. The mass of the atoms involved in each bond is $m_{a t}$, and $I$ is the lattice parameter [18] [19]. The values of $m_{a t}$ and $l$ are displayed in Table 1. For the rhombohedral $\mathrm{LaAlO}_{3}$ structure, which is non-cubic, we choose an average value for $l$, reported in Table 1 . The values of the vibrational frequency $\omega$ of the bond derived as $w_{c}$, i.e. the location of the minima of the absorption bands in the FTIR spectra in Figure 1, Figure 2, and Figure 3, appear in Table 1 and Table 2. We claim that Equation (2) explains the change with $P$ of the values of $\omega$, and believe that the complex interaction among the vibrations or rotations of the bonds illuminated by the IR light justifies the observation that these changes are not necessarily monotonic. This issue however requires further investigations.

Finally, in Equation (2), the number $\eta$ represents the number of bonds involved in a vibration. The need to introduce the number $\eta$ can be explained as follows. During the absorption of the IR light, when the average power $P$ changes from $7.9 \mathrm{~mW}$, to $12.1 \mathrm{~mW}$, to $16.4 \mathrm{~mW}$ or to $25 \mathrm{~mW}$ or vice versa, the moment of inertia $I$ does not change. Simultaneously, the magnitude of the vibrational frequency $\omega$ of each bond is constrained by the corresponding experimentally measured values. Therefore, a degree of freedom is required to match the energy absorbed from the IR light by the crystalline thin film, to the magnitude of the energy transferred from the IR light. The number $\eta$ is such a degree of freedom. Equation (2) suggests that larger values of $P$ and $\tau$ increase the energy transferred from the IR light to the thin films and, by doing so, involve a larger number of bonds $\eta$. This trend is confirmed by the estimated values of $\eta$ for both the cubic $\mathrm{Yb}_{2} \mathrm{O}_{3}$ and rhombohedral $\mathrm{LaAlO}_{3}$ films on Si reported in Table 3 and Table 4.

We can extract further consequences from Equation (2). One is that the direct proportionality between the moment of inertia $I$ of each bond and the average power $P$ of the IR light, i.e. $I \propto P$, suggests that FTIR spectrometers with larger values of $P$ than the current ones might generate spectra highly sensitive to the features of crystalline films with heavy atoms (low $\omega$ ) in the far IR region (smaller $\tau$ ). The second consequence is that Equation (2) might foster the understanding of phonon's behavior in crystals. Indeed, the direct proportionality between the number of bonds and the average power, i.e. $\eta \propto P$, suggests that the number of phonons increases with the average power of the IR light. Thus, 
Table 3. Vibrational energy $E_{v i b}$ of the crystal lattices of cubic $\mathrm{Yb}_{2} \mathrm{O}_{3}$ and rhombohedral $\mathrm{LaAlO}_{3}$ at the minima of the absorption bands at vibrational frequencies $\omega$, and the number of bonds $\eta$ involved in the vibrations at $P=25 \mathrm{~mW}$ and $12.1 \mathrm{~mW}$ of the illuminating IR light over a surface area of $\sim 1 \mathrm{~cm}^{2}$. The values of $\omega$ are extracted from Figure 1 and are the parameter $w_{c}$ in Equation (1).

\begin{tabular}{ccc}
\hline $\mathrm{Yb}_{2} \mathrm{O}_{3}$ on Si & $E_{\text {vib }}(\mathrm{aJ})$ & $\eta$ \\
\hline $25 \mathrm{~mW}$ & 98.0 & 9.88 \\
$12.1 \mathrm{~mW}$ & 98.54 & 4.84 \\
$25 \mathrm{~mW}$ & 105.81 & 8.80 \\
$12.1 \mathrm{~mW}$ & 106.86 & 4.28 \\
$25 \mathrm{~mW}$ & 118.31 & 7.45 \\
$12.1 \mathrm{~mW}$ & 118.06 & 3.69 \\
$\mathrm{LaAlO}$ on Si & & \\
$25 \mathrm{~mW}$ & 10.41 & 160.95 \\
$12.1 \mathrm{~mW}$ & 10.45 & 79.0 \\
$25 \mathrm{~mW}$ & 12.38 & 124.14 \\
$12.1 \mathrm{~mW}$ & 12.36 & 61.44 \\
\hline
\end{tabular}

Table 4. Vibrational energy $E_{v i b}$ of the crystal lattices of cubic $\mathrm{Yb}_{2} \mathrm{O}_{3}$ and rhombohedral $\mathrm{LaAlO}_{3}$ at the minima of the absorption bands at vibrational frequencies $\omega$, and the number of bonds $\eta$ involved in the vibrations at $P=25 \mathrm{~mW}, 16.4 \mathrm{~mW}$ and $7.9 \mathrm{~mW}$ of the illuminating IR light over a surface area of $\sim 1 \mathrm{~cm}^{2}$. The values of $\omega$, or the parameter $w_{c}$ in Equation (1), are extracted from Figure 2 for the $\mathrm{Yb}_{2} \mathrm{O}_{3}$ films and from Figure 3 for the $\mathrm{LaAlO}_{3}$ films. For simplicity for the $\mathrm{Yb}_{2} \mathrm{O}_{3}$ films we have chosen only two out of the three relevant absorption bands.

\begin{tabular}{ccc}
\hline $\mathrm{Yb}_{2} \mathrm{O}_{3}$ on Si & $E_{\text {vib }}(\mathrm{aJ})$ & $\eta$ \\
$25 \mathrm{~mW}$ & 97.91 & 9.69 \\
$16.4 \mathrm{~mW}$ & 97.92 & 6.62 \\
$7.9 \mathrm{~mW}$ & 97.95 & 3.12 \\
$25 \mathrm{~mW}$ & 118.13 & 7.31 \\
$16.4 \mathrm{~mW}$ & 118.31 & 4.98 \\
$7.9 \mathrm{~mW}$ & 118.26 & 2.40 \\
$\mathrm{LaAlO}$ on Si & & \\
$25 \mathrm{~mW}$ & 10.42 & 157.51 \\
$16.4 \mathrm{~mW}$ & 10.40 & 107.93 \\
$7.9 \mathrm{~mW}$ & 10.44 & 51.67 \\
$25 \mathrm{~mW}$ & 12.37 & 121.70 \\
$16.4 \mathrm{~mW}$ & 12.38 & 83.05 \\
$7.9 \mathrm{~mW}$ & 12.36 & 40.13 \\
\hline
\end{tabular}

the number of phonons involved in the interaction between IR light and a thin crystalline film is constrained by the magnitude of the average power $P$ of the IR light, not just by the scattering events occurring between phonons and imperfec- 
tions in crystalline thin films.

Experimental results of other authors help us to assess the validity of Equation (2). We will present two examples: one from Faulkner et al. [20], and another by Reimann et al. [9].

Faulkner et al. [20] investigated the effects of a laser beam on the $E_{v i b}$ of $\mathrm{He}$ nano-droplets at $9 \mathrm{~K}$. These authors used an IR laser with an average power $P$ of $\sim 80 \mathrm{~mW}$, and observed a resonance at $2000 \mathrm{~cm}^{-1}\left(6 \times 10^{13} \mathrm{~Hz}\right.$ or $\left.16.7 \mathrm{fs}\right)$ with a ro-vibrational transition in a carbonyl sulphide (OCS) molecule accommodated in a He nano-droplet. Furthermore, the transfer of energy from the IR laser to the OCS molecule was observed to lead to the evaporation of $\sim 400 \mathrm{He}$ atoms in the nano-droplet. Using Equation (2) we estimate the energy transferred from the IR laser to the OCS molecule to be $P \tau=1.3 \times 10^{-15} \mathrm{~J} \quad$ (or $8.3 \mathrm{keV}$ ). The OCS molecule uses this energy to initiate a ro-vibrational motion which, in turn, transfers an energy of $\sim 3.3 \times 10^{-18} \mathrm{~J}$ (or $20.8 \mathrm{eV}$ ) to each of the $400 \mathrm{He}$ atoms in the nano-droplet for a $2000 \mathrm{~cm}^{-1}$ excited state. The $20.8 \mathrm{eV}$ for each He atom is about $25 \times 10^{3}$ times larger than the $0.83 \mathrm{meV}$ energy per atom estimated to enable the evaporation of the He atoms (P. L. Raston, James Madison University, oral communication, 2018). We justify this four orders of magnitude discrepancy between the evaporation energies per atom, $20.8 \mathrm{eV}$ and $0.83 \mathrm{meV}$, by claiming that $20.8 \mathrm{eV}$ for each $\mathrm{He}$ atom are needed to overcome the strong coupling among the $\mathrm{He}$ atoms in superfluid $\mathrm{He}$ nanoparticles at low temperature $(9 \mathrm{~K})$. On the other hand, the evaporation energy per He atom of $0.83 \mathrm{meV}$, estimated by the authors of Ref. [20], seems very small. Indeed, for comparison, we recall that an energy of $\sim 50 \mathrm{meV}$ transmitted by visible light with $P \sim \mu W$ to electrons in semiconductors is sufficient to cause each one of them to jump from shallow donor levels to the conduction band [21]. This amount of energy is small but consistent with the fact that the mass of one electron is about four orders of magnitude smaller than the mass of a He atom.

Reimann et al. [9] investigated the relationship between light's power $P$ and the rotational frequency $\omega$ of an optically trapped silica nanoparticle [9]. In their experiment, a laser beam illuminates a nanoparticle, transfers energy to it, and causes the nanoparticle to rotate. From Equation (2), we estimate that, with a laser beam with wavelength $1565 \mathrm{~nm}$ ( $\tau=5.2 \mathrm{fs}$ ) and average power $P$ of $200 \mathrm{~mW}$ [9], the energy transferred to the nanoparticle is $P \tau=1.04 \mathrm{fJ}$. To induce nanoparticle to rotate with rotational frequency $\omega=1.31 \mathrm{GHz}$ at a pressure of $1.1 \times 10^{-5}$ mbar, this value of $P \tau$ requires a moment of inertia $I=1.21 \times 10^{-33} \mathrm{~kg} \cdot \mathrm{m}^{2}$. From this value of $I$, and knowing that the spherical nanoparticle's radius is 50 $\mathrm{nm}$ [9], we infer that the mass of one silica nanoparticle is $0.968 \times 10^{-18} \mathrm{~kg}$, which implies a density of $1.85 \times 10^{3} \mathrm{~kg} / \mathrm{m}^{3}$. Such a density is only 3.7 times the published value of $0.49 \times 10^{3} \mathrm{~kg} / \mathrm{m}^{3}$ [22]. This finding suggests that $P \tau=1.04 \mathrm{fJ}$ is a good estimate of the energy transferred from the IR light to the nanoparticle. This finding also enables us to exploit Equation (2) to obtain the rotational frequency $\omega$ versus average power $P$ in the case of $\eta=1$ at a pressure of $\sim 1.1 \times$ $10^{-5} \mathrm{mbar}$, and $I=1.21 \times 10^{-33} \mathrm{~kg} \cdot \mathrm{m}^{2}$. The result is reported in Figure 5 , which 


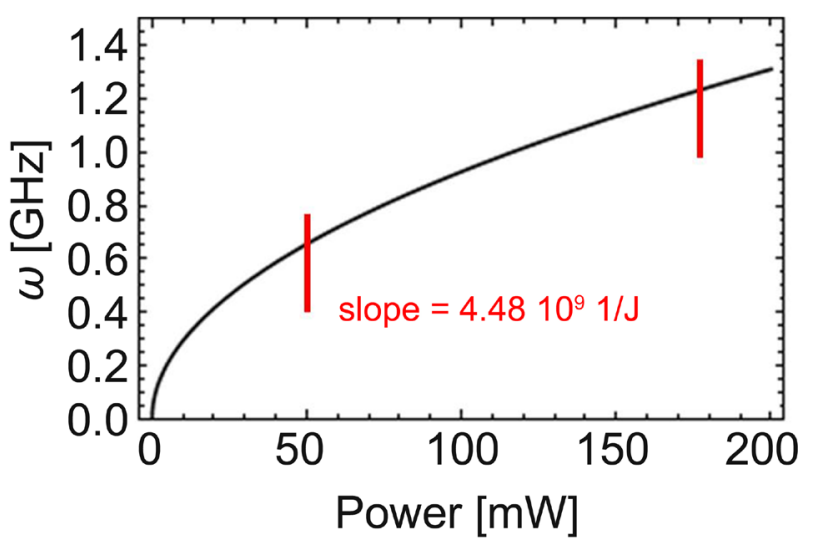

Figure 5. Rotational frequency $\omega$ of a nanoparticle versus average power $P$ of the IR light obtained using Equation (2) with average power $200 \mathrm{~mW}$ at a wavelength of 1565 $\mathrm{nm}(\tau=5.2 \mathrm{fs})$ from Ref. [9], $P \tau=1.04 \mathrm{fJ}, I=1.21 \times 10^{-33} \mathrm{~kg} \cdot \mathrm{m}^{2}$, and $\eta=1$, a for nanoparticles at a pressure of $1.1 \times 10^{-5}$ mbar. The rotational frequency $\omega$ increases almost linearly with $P$ at a "slope" of $4.48 \times 10^{9} \mathrm{~J}^{-1}$ between the two bars. This finding is in good agreement with Ref. [9], which reports a "slope" of $0.3 \times 10^{9} \mathrm{~J}^{-1}$ at a pressure of $1 \times$ $10^{-4}$ mbar.

shows that the rotational frequency $\omega$ increases almost linearly with the average power $P$ between the two bars with a "slope" of $4.48 \times 10^{9} \mathrm{~J}^{-1}$. This finding is in good agreement with the "slope" of $0.3 \times 10^{9} \mathrm{~J}^{-1}$ at a pressure of $1 \times 10^{-4} \mathrm{mbar}$ found in Ref. [9]. This result reveals the relationship between $P$ and $\omega$ of an optically trapped silica nanoparticle [9], and pictures the torque transfer mechanism involved in this experiment as an effect of the law of conservation of energy.

\section{Conclusions}

Our work proposes a case in which the magnitude of the average power $P$ of an electromagnetic (EM) wave, together with its period $\tau$, may be correlated with the magnitude of the mechanical motions that either generate the EM waves or are generated by them. Using Fourier transform infrared (IR) spectra of thin films we have shown that the average power $P$ of the IR light affects the mechanical motion, in the form of rotation or vibration, of the bonds involved. We explain the phenomenon by linking, through the law of conservation of energy, the value of the energy $P \tau$, where $\tau$ is the period of the IR light, to the vibrational frequency $\omega$, the number $\eta$, and the moment of inertia $I$ of the rotating or vibrating bonds affected by the illumination under the IR light. From a practical point of view, this finding highlights the possibility of manufacturing IR spectrometers adopting sources of IR light with larger average power $P$ than those currently used. Such a strategy would enable maximizing the sensitivity of the Fourier transform IR spectra of crystalline films with heavy atoms in the far IR region. The users of spectrometers with higher power IR light sources should however be aware of the shifts in location of the minima of the absorption bands arising with changing $P$. From a more speculative point of view, our finding 
highlights that, as the magnitude of $P \tau$ increases, so does the number $\eta$ of the crystal bonds involved in rotational or vibrational motions. The number $\eta$ represents a "classical" way (as opposed to the "quantum" way) to count the number of the quasi-particles usually called phonons through the direct relationship between the energy $P \tau$ with the vibrational frequency $\omega$ and the moment of inertia $I$ of the rotating or vibrating bonds affected by the illumination under the IR light. Thus, since the number $\eta$ can be viewed as the number of phonons in a crystal lattice, we conclude that the number $\eta$ of phonons is constrained by the average power $P$ and the period $\tau$ of the IR light impinging on a thin crystalline film, not only by phonon scattering phenomena.

Finally, in the context of the current scientific literature our findings are relevant because they either justify results otherwise not explained, or agree with other reports. For instance, our results justify the displacement in the minima of the absorption bands in Fourier transform IR spectra detected but not explained by other authors [14]. In addition, our model based on the law of conservation of energy agrees qualitatively and/or quantitatively with results presented in the current literature regarding Thomson scattering [3] [4], neutron star merger events [7], vibrational spectroscopy [2] [20] and optical manipulation of nanoparticles [9].

\section{Acknowledgements}

This work was supported by the U.S. Office of Naval Research (ONR) awards \# N000141410378 and N000141512158, the JMU 4-VA Consortium (2016-2017), the JMU Center for Materials Science, and the JMU Department of Physics and Astronomy. The authors thank Prof. Gregory N. Parsons (NCSU) and Dr. John C. Zimmerman (JMU) for fruitful discussions.

\section{Conflicts of Interest}

The authors declare no conflicts of interest regarding the publication of this paper.

\section{References}

[1] Garcia-López, V., Chen, F., Nilawski, L.G., Duret, G., Aliyan, A., Kolomeisky, A.B., Robinson, J.T., Wang, G., Pal, R. and Tour, J.M. (2017) Molecular Machines Open Cell Membranes. Nature, 548, 567-572. https://doi.org/10.1038/nature23657

[2] Stensitzki, T., Yang, Y., Kozich, V., Ahmed, A.A., Kössl, F., Kühn, O. and Heyne, K. (2018) Acceleration of a Ground-State Reaction by Selective Femtosecond-InfraredLaser-Pulse Excitation. Nature Chemistry, 10, 126-131. https://doi.org/10.1038/nchem.2909

[3] Yan, W., Fruhling, C., Golovin, G., Heden, D., Luo, J., Zhang, P., Zhao, B., Zhang, J., Liu, C., Chen, M., Chen, S., Banerjee, S. and Umstadter, D. (2017) High-Order Multiphoton Thomson Scattering. Nature Photonics, 11, 514-520. https://doi.org/10.1038/nphoton.2017.100

[4] Har-Shemesh, O. and Di Piazza, A. (2012) Peak Intensity Measurement of Relativistic Lasers via Nonlinear Thomson Scattering. Optics Letters, 37, 1352-1534. 
https://doi.org/10.1364/OL.37.001352

[5] Xu, Y., Cao, W., Ahn, K., Jing, J., Chae, J., Huang, N., Deng, N., Gary, D.E. and Wang, H. (2018) Transient Rotation of Photospheric Vector Magnetic Fields Associated with Solar Flare. Nature Communications, 9, 1-8. https://doi.org/10.1038/s41467-017-02509-w

[6] Smit, R., Bouwens, R.J., Carniani, S., Oesch, P.A., Labbé, I., Illingworth, G.D., Van Der Werf, P., Bradley, L.D., Gonzalez, V., Hodge, J.A., Holwerda, B.W., Maiolino, R. and Zheng, W. (2018) Rotation in [CII]-Emitting Gas in Two Galaxies at a Redshift of 6.8. Nature, 553, 178-181. https://doi.org/10.1038/nature24631

[7] Mooley, K.P., Nakar, E., Hotokezaka, K., Hallinan, G., Corsi, A., Frail, D.A., Horesh, A., Murphy, T., Lenc, E., Kaplan, D.L., De, K., Dobie, D., Chandra, P., Deller, A., Gottlieb, O., Kasliwal, M.M., Kulkarni, S.R., Myers, S.T., Nissanke, S., Piran, T., Lynch, C., Bhalerao, V., Bourke, S., Bannister, K.W. and Singer, L.P. (2018) A Mildly Relativistic Wide-Angle Outflow in the Neutron-Star Merger Event GW170817. Nature, 554, 207-210. https://doi.org/10.1038/nature25452

[8] Draine, B.T. and Lazarian, A. (1998) Electric Dipole Radiation from Spinning Dust Grains. The Astrophysical Journal, 508, 157-179. https://doi.org/10.1086/306387

[9] Reimann, R., Doderer, M., Hebestreit, E.E., Diehl, R., Frimmer, M., Windey, D., Tebbenjohanns, F. and Novotny, L. (2018) GHz Rotation of an Optically Trapped Nanoparticle in Vacuum. Physical Review Letters, 121, 1-5.

[10] Boone, D.E., Jackson, C.H., Swecker, A.T., Hergenrather, J.S., Wenger, K.S., Kokhan, O., Terzić, B., Melnikov, I., Ivanov, I.N., Stevens, E.C. and Scarel, G. (2018) Probing the Wave Nature of Light-Matter Interaction. World Journal of Condensed Matter Physics, 8, 62-89. https://doi.org/10.4236/wjcmp.2018.82005

[11] Griffiths, P.R. and de Haseth, J.A. (2007) Fourier Transform Infrared Spectrometry. John Wiley \& Sons, Hoboken, NJ.

[12] Parsons, G.N., George, S.M. and Knetz, M. (2011) Progress and Future Directions for Atomic Layer Deposition and ALD-Based Chemistry. MRS Bulletin, 36, 865-871. https://doi.org/10.1557/mrs.2011.238

[13] Scarel, G., Svane, A. and Fanciulli, M. (2007) Rare Earth Oxide Thin Films: Growth, Characterization, and Applications. In: Fanciulli, M. and Scarel, G., Eds., Topics in Applied Physics, Springer Verlag, Heidelberg, Vol. 106.

[14] Scarel, G., Hirschmugl, C.J., Yakovlev, V.V., Sorbello, R.S., Aita, C.R., Tanaka, H. and Hisano, K. (2002) Infrared Response of Vitreous Titanium Dioxide Films with Anatase Short-Range Order. Journal of Applied Physics, 91, 1118-1128. https://doi.org/10.1063/1.1427430

[15] Malvestuto, M., Scarel, G., Wiemer, C., Fanciulli, M. and D’Acapito, F. (2006) X-Ray Absorption Spectroscopy Study of $\mathrm{Yb}_{2} \mathrm{O}_{3}$ and $\mathrm{Lu}_{2} \mathrm{O}_{3}$ Thin Films Deposited on $\mathrm{Si}(100)$ by Atomic Layer Deposition. Nuclear Instruments and Methods in Physics Research Section B, 246, 90-95. https://doi.org/10.1016/j.nimb.2005.12.020

[16] Pavlik III, A., Ushakov, S.V., Navrotsky, A., Benmore, C.J. and Weber, R.J.K. (2017) Structure and Thermal Expansion of $\mathrm{Lu}_{2} \mathrm{O}_{3}$ and $\mathrm{Yb}_{2} \mathrm{O}_{3}$ up to the Melting Points. Journal of Nuclear Materials, 495, 385-391.

[17] Sanz-Ortiz, M.N., Rodríguez, F., Rodríguez, J. and Demazeau, G. (2011) Optical and Magnetic Characterisation of $\mathrm{Co}^{3+}$ and $\mathrm{Ni}^{3+}$ in $\mathrm{LaAlO}_{3}$ : Interplay between the Spin State and Jahn-Teller Effect. Journal of Physics: Condensed Matter, 23, Article ID: 415501. https://doi.org/10.1088/0953-8984/23/41/415501

[18] Adachi, G.-Y. and Imanaka, N. (1998) The Binary Rare Earth Oxides. Chemical Re- 
views, 98, 1479-1514.

[19] Zaho, J., Angel, R.J. and Ross, N.L. (2011) The Structural Variation of Rhombohedral $\mathrm{LaAlO}_{3}$ Perovskite under Non-Hydrostatic Stress Fields in a Diamond-Anvil Cell. Journal of Physics: Condensed Matter, 23, Article ID: 175901.

[20] Faulkner, T., Miller, I. and Raston, P.L. (2018) Quantum Cascade Laser Spectroscopy of OCS Isotopologues in ${ }^{4} \mathrm{He}$ Nanodroplets: A Test of Adiabatic Following for a Heavy Rotor. The Journal of Chemical Physics, 148, Article ID: 044308. https://doi.org/10.1063/1.5009908

[21] Sarker, B.K., Cazalas, E., Chung, T.-F., Childres, I., Jovanovic, I. and Chen, Y.P. (2017) Position-Dependent and Millimetre-Range Photodetection in Phototransistors with Micrometre-Scale Graphene on SiC. Nature Nanotechnology, 12, 668-674.

[22] Kimoto, S., Dick, W.D., Syedain, Z., Pui, D.Y.H. and Roberts, D.L. (2014) Effective Density of Silica Nanoparticle Size Standards. 2014 International Aerosol Conference, Busan, 28 August-2 September 2014.

[23] Scarel, G., Bonera, E., Wiemer, C., Tallarida, G., Spiga, S., Fanciulli, M., Fedushkin, I.L., Schumann, H., Lebedinskii, Y. and Zenkevich, A. (2004) Atomic-Layer Deposition of $\mathrm{Lu}_{2} \mathrm{O}_{3}$. Applied Physics Letters, 85, 630-632.

[24] Bonera, E., Scarel, G., Fanciulli, M., Delugas, P. and Fiorentini, V. (2005) Dielectric Properties of High- $\kappa$ Oxides: Theory and Experiment for $\mathrm{Lu}_{2} \mathrm{O}_{3}$. Physical Review Letters, 94, Article ID: 027602.

[25] Gordon, A.L. and Scarel, G. (2018) Interaction in the Steady State between Electromagnetic Waves and Matter. World Journal of Condensed Matter Physics, 8, 171-183.

[26] St. John, T.C., Marinelli, Z.J., Kaczmar, J.M., Given, R.P., Wenger, K.S., Utter, B.C. and Scarel, G. (2016) Conversion of Infrared Light into Usable Energy. Proceedings of SPIE, 9927, 99270C. 


\section{Appendix 1. Crystallinity of the Thin Films}

We studied the crystallinity of the thin films on silicon using Fourier transform infrared (FTIR) spectroscopy in the far infrared (IR) region at $0^{\circ}$ and $70^{\circ}$ angle of incidence. Figure A1(a) shows the far-IR FTIR spectra of cubic structure of the $\mathrm{Yb}_{2} \mathrm{O}_{3}$ films [23] [24]. Figure $\mathrm{A} 1(\mathrm{~b})$ reports the rhombohedral structure of the far-IR FTIR spectra of $\mathrm{LaAlO}_{3}$ films on Si: this result is reported in G. Scarel, C. Wiemer, S. Spiga, M. Fanciulli, A. Zenkevich and Y. Lebedinskii, MDM National Laboratory-Italy, unpublished results, 2007.

\section{Appendix 2. Wide FTIR Spectra with Double Side Polished Si Substrate}

For the measurements presented in the main text we used single side polished $\mathrm{Si}$ as substrate for the thin films and double side polished $\mathrm{Si}$ as reference for the FTIR measurements. In Figure A2 we show the spectra on a larger wavenumber range compared to the range used in Figure 2(a) and Figure 3(a) of the main text. Figure A2(a) and Figure A2(b) show the original FTIR spectra of cubic $\mathrm{Yb}_{2} \mathrm{O}_{3}$ films on $\mathrm{Si}$ acquired with IR light's power of $7.9 \mathrm{~mW}$ and $25 \mathrm{~mW}$ over a broad (a) and a more restricted (b) spectral range. The restricted range is closer to the far-IR region where the typical absorption bands of cubic $\mathrm{Yb}_{2} \mathrm{O}_{3}$ are found. Panels (c) and (d) show the original transmission FTIR spectra of rhombohedral $\mathrm{LaAlO}_{3}$ films on $\mathrm{Si}$ acquired with IR light's power of $7.9 \mathrm{~mW}$ and $25 \mathrm{~mW}$. Also in this case the restricted spectral range in panel (d) is closer to the far-IR region where the typical absorption bands of rhombohedral $\mathrm{LaAlO}_{3}$ are found.

\section{Appendix 3. Wide FTIR Spectra with Single Side Polished Si Substrate}

For comparison, we performed some measurements with single side Si a reference. This Si reference is similar to the Si substrate on which the thin films were deposited. We call this substrate as $\mathrm{Si}(2)$. Figure A3(a) and Figure A3(b) show the original FTIR spectra of cubic $\mathrm{Yb}_{2} \mathrm{O}_{3}$ films on $\mathrm{Si}(2)$ acquired with IR light's power of $16.4 \mathrm{~mW}$ and $25 \mathrm{~mW}$ over a broad (a) and a more restricted (b) spectral range closer to the far-IR region where the absorption bands of cubic $\mathrm{Yb}_{2} \mathrm{O}_{3}$ are found. The background is different compared to the one in Figure A2(a)
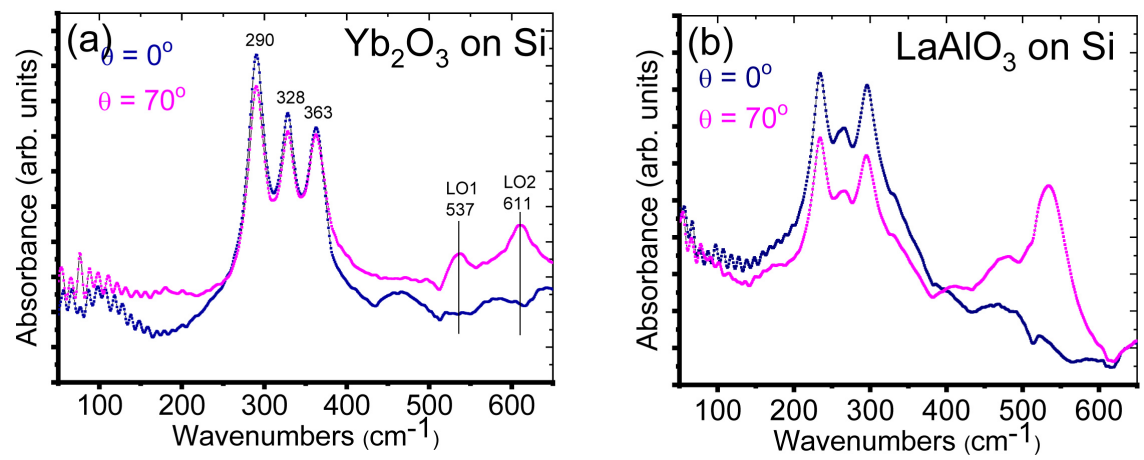

Figure A1 

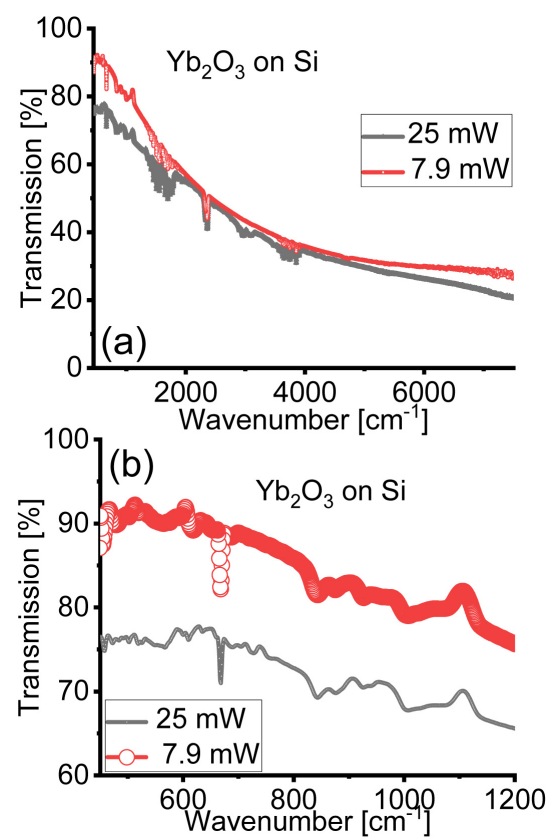
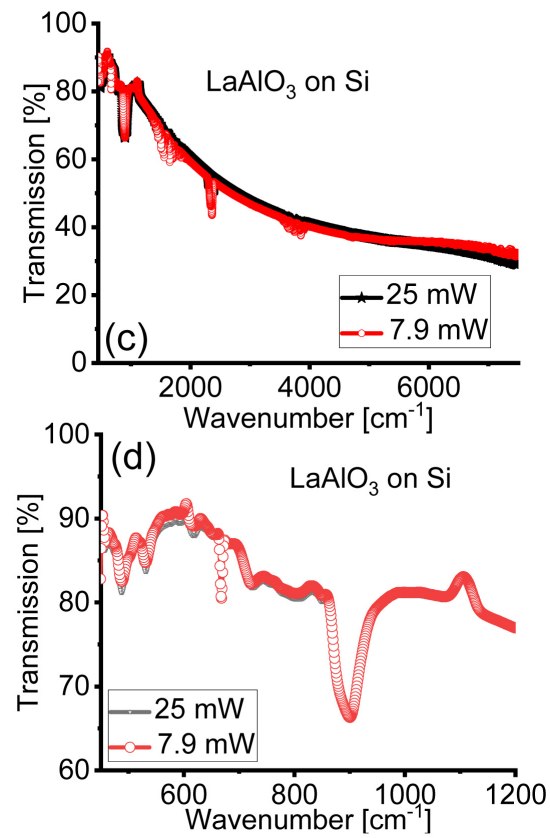

Figure A2
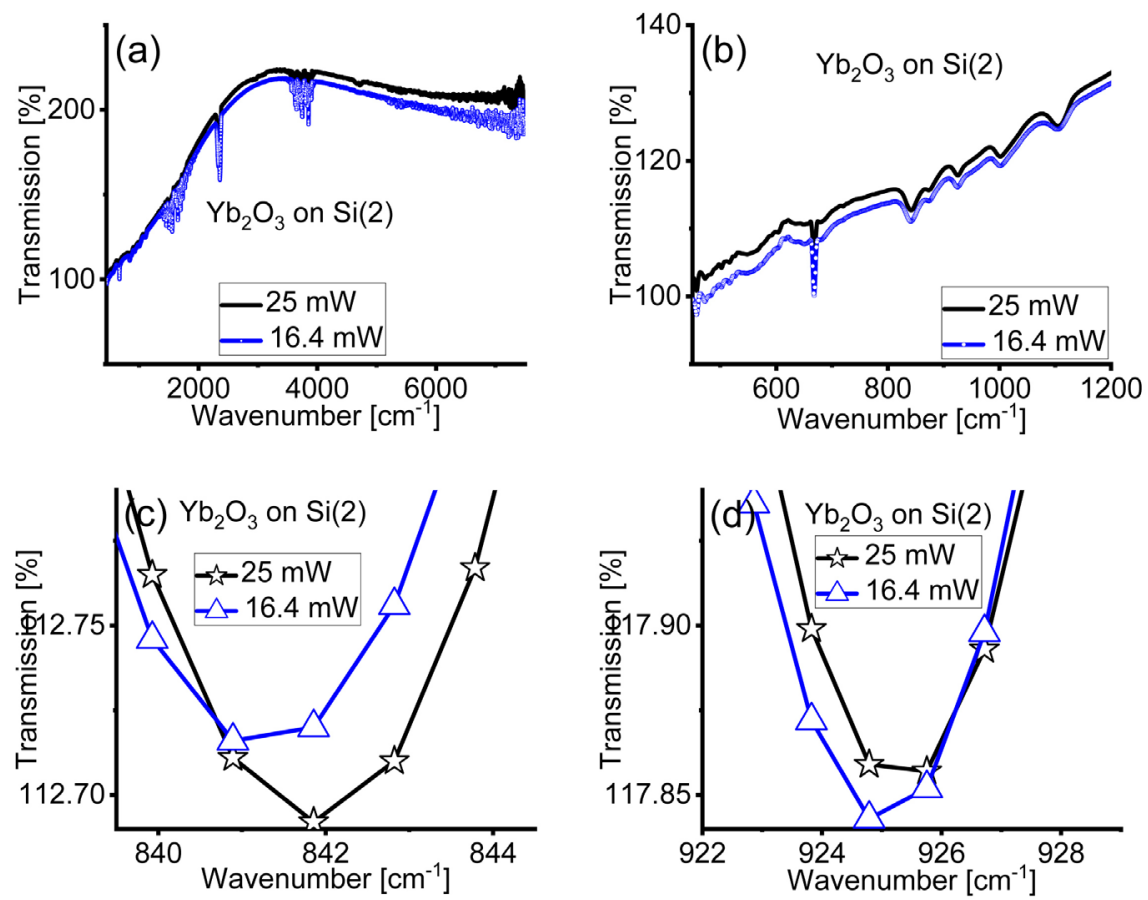

Figure A3

and Figure A2(b), however the absorption bands are the same. Panels (c) and (d) zoom into the range of the absorption bands of the $\mathrm{Yb}_{2} \mathrm{O}_{3}$ films. These absorption bands are the same as in Figure 2(b) and Figure 2(c) in the main text. We observe a displacement of the absorption bands with the different value of average power of the IR light. All the absorption bands are slightly shifted toward lower wavenumbers with $\mathrm{Si}(2)$. We conclude that the displacements of the ab- 
sorption bands do not depend on the Si used as reference.

Figure A4(a) and Figure A4(b) show the original FTIR spectra of rhombohedral $\mathrm{LaAlO}_{3}$ films on $\mathrm{Si}(2)$ acquired with IR light's power of $16.4 \mathrm{~mW}$ and 25 $\mathrm{mW}$ over a broad (a) and a more restricted (b) spectral range closer to the far-IR region where the absorption bands of rhombohedral $\mathrm{LaAlO}_{3}$ are found. The background is different compared to the one in Figure A2(c) and Figure A2(d), however the absorption bands are the same. Panels (c) and (d) zoom into the range of the absorption bands of the $\mathrm{LaAlO}_{3}$ films. These absorption bands are the same as in Figure 3(b) and Figure 3(c) in the main text. We observe a displacement of the absorption bands with the different value of average power of the IR light. All the absorption bands are slightly shifted toward lower wavenumbers with $\mathrm{Si}(2)$.

\section{Appendix 4. Can We Find Displacements in the Location of the Absorption Bands of the Molecular Vibrations?}

We tested whether molecular vibrations are affected by the power of the IR light using the same spectra we obtained for the measurements discussed in the main text collected with the Bruker spectrometer with IR light at average power $P=$ $7.9 \mathrm{~mW}$ and $P=25 \mathrm{~mW}$ for $\mathrm{Yb}_{2} \mathrm{O}_{3}$ films on $\mathrm{Si}$ and $\mathrm{LaAlO}_{3}$ films on Si. The relevant results are shown in Figure A5. In panels (a) and (c) we zoom into the 2,500 $\mathrm{cm}^{-1}$ region where carbon residues are found. A close inspection in the 2,361 $\mathrm{cm}^{-1}$ region in panels (b) and (d) indicates no displacement in the location of the minima for the two different values of the average power. We notice however different depth in the absorption bands and ascribe the effect to the slight different purge time before collecting the spectra. Because of this finding we infer
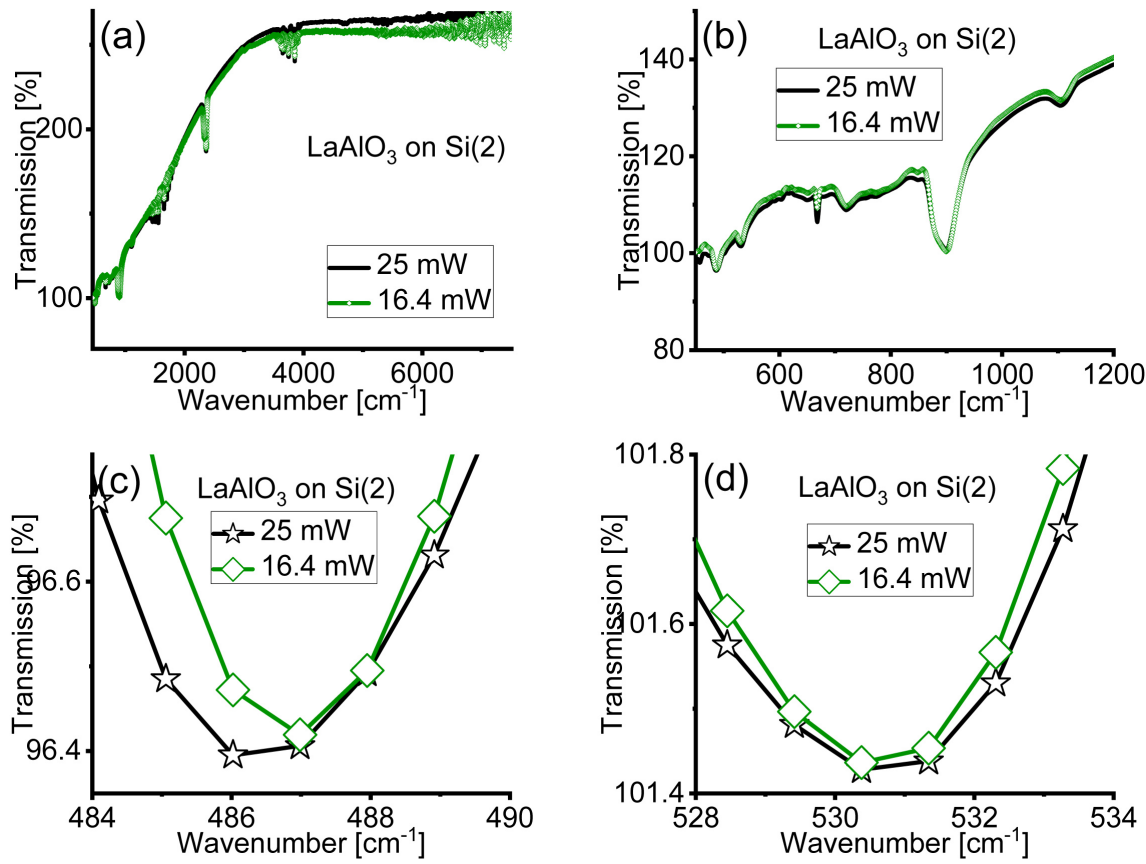

Figure A4 

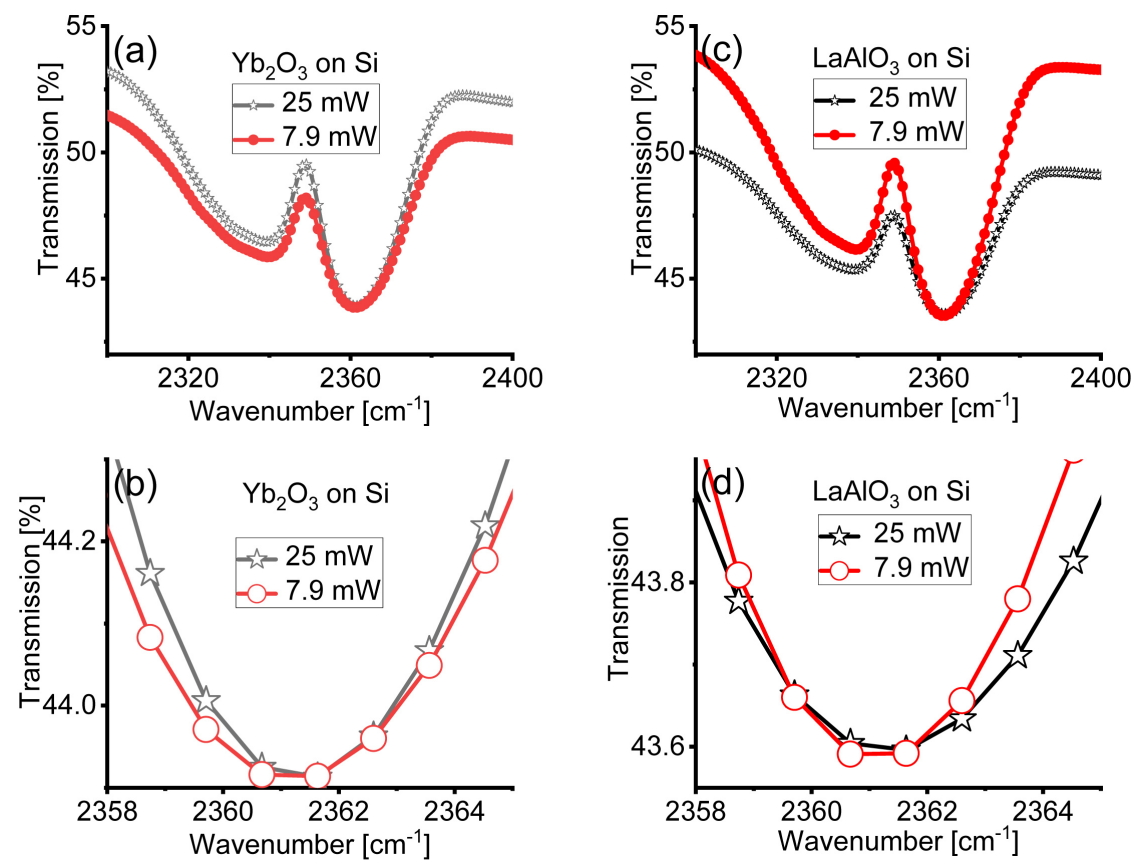

Figure A5

that molecular vibrations are not affected by the values of the IR light power as the crystalline bonds. A more focused research on the effects of the power of the IR light on molecules would be necessary understand the issue in a more complete manner.

\section{Appendix 5. Can We Find Displacements in the Location of the Minima of the Absorption Bands Due to Sample Heating?}

We performed temperature measurements by placing a thermocouple on the sample exactly where the IR beam hits the sample. In Figure A6(a) we show the jump in temperature observed on a sample in the first 400 seconds after starting the illumination with the $25 \mathrm{~mW}$ IR beam of the Bruker spectrometer. The amplitude of the jump is $0.035^{\circ} \mathrm{C}$. In Figure A6(b) we show the jump in temperature observed on a sample in the first 400 seconds after starting the illumination with the $7.9 \mathrm{~mW}$ IR beam of the Bruker spectrometer with the polarizer in the IR beam path. The amplitude of the jump is $0.03^{\circ} \mathrm{C}$. We consider the $0.035^{\circ} \mathrm{C}$ and $0.03^{\circ} \mathrm{C}$ differences in amplitude of the temperature jumps as negligible and therefore ineffective in causing the displacements in the location of the minima of the absorption bands. The results of the temperature measurements, summarized in Figure A6, are partly included in Refs. [10] [25] and [26].

\section{Appendix 6. Assessment of the Reproducibility of the Location of the Minima of the Absorption Bands for the Rhombohedral $\mathrm{LaAlO}_{3}$ Films on Si.}

In Figure A7 we show the FTIR spectra of the rhombohedral $\mathrm{LaAlO}_{3}$ films on $\mathrm{Si}$ acquired with IR light's power of $25 \mathrm{~mW}$ (panels (a) and (c)) and $7.9 \mathrm{~mW}$ (panels 

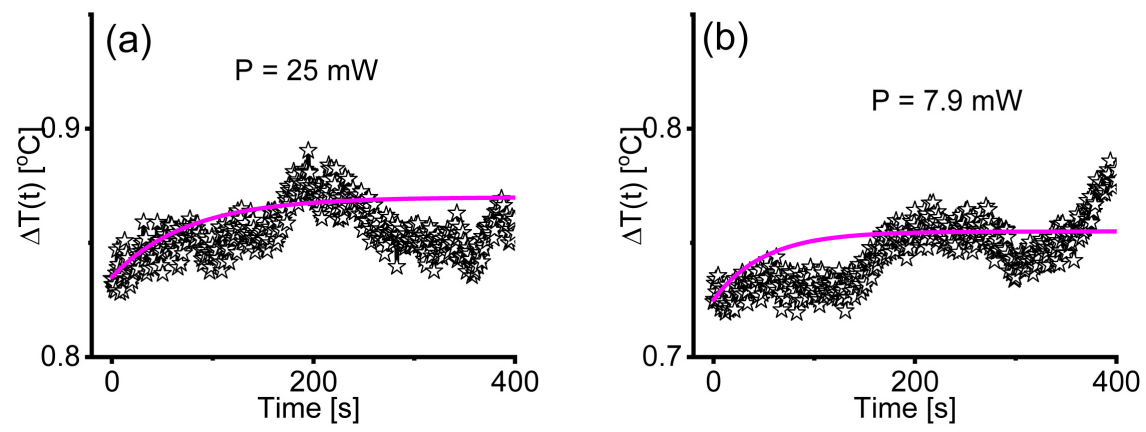

Figure A6
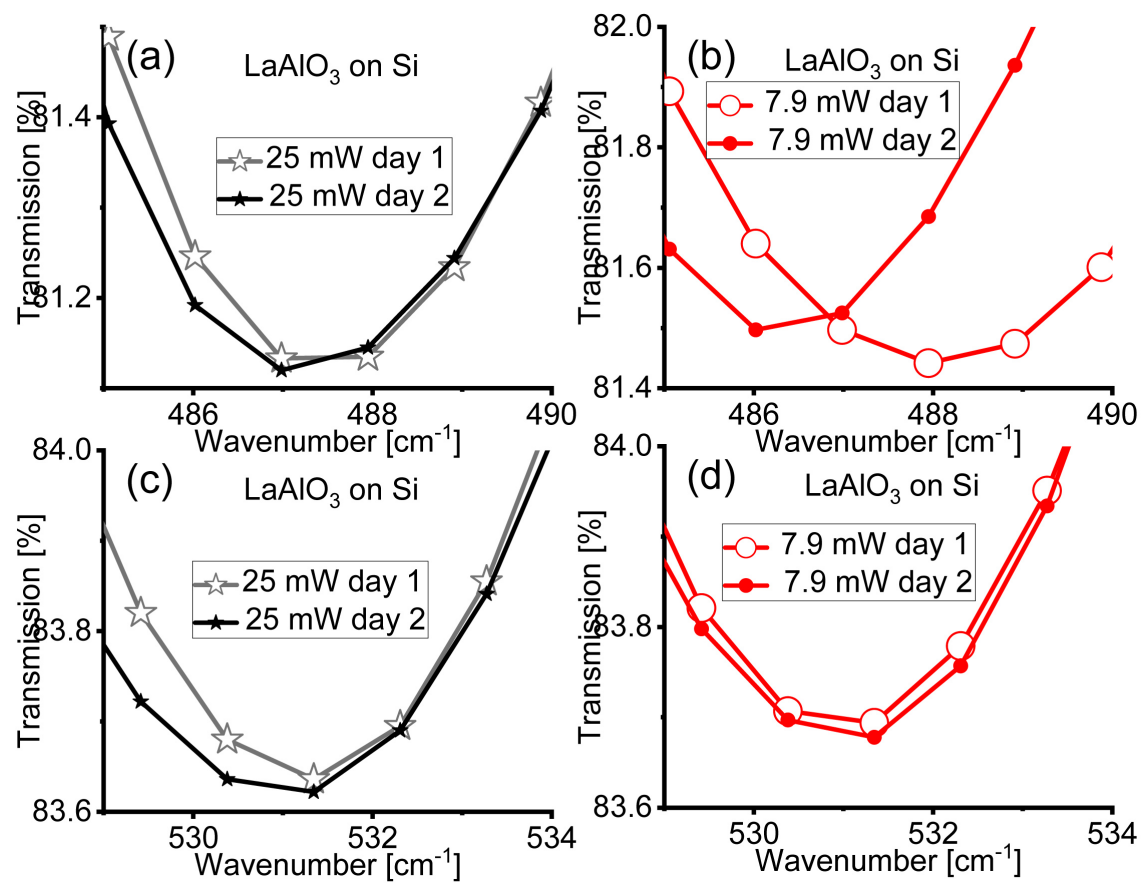

Figure A7

(b) and (d)) in two different days (day 1 and day 2). Most of the absorption bands do not change their location in different days. However a significant displacement is observed in the far-IR region for the low power case. We ascribe the effect to a random interaction among the vibrations or rotations of the bonds. 